\title{
Double-balloon enteroscopy-assisted ERCP in situs inversus with Roux-en-Y hepaticojejunostomy for complex anastomotic stricture dilated with Soehendra stent retriever
}

A woman in her 50 s with situs inversus presented to our hospital with high fever, jaundice, and pain in the left hypochondriac region. The patient had undergone Roux-en-Y hepaticojejunostomy following iatrogenic bile duct injury during cholecystectomy 9 years earlier. Abdominal computed tomography and magnetic resonance cholangiopancreatography revealed bile duct dilatation, with areas suspicious for stenosis at the hepaticojejunostomy anastomosis, with the visceral organs in a mirror image location from their normal positions ( $\mathbf{F i g . 1 ,}$ - Fig. 2). We diagnosed obstructive jaundice with cholangitis, and performed endoscopic retrograde cholangiopancreatography (ERCP) for biliary drainage using double-balloon enteroscopy (EI-580BT; Fujifilm, Tokyo, Japan).

The patient was placed in the abdominal position, with the endoscopist standing at the patient's right side, which is the standard position for ERCP. Endoscope insertion to the hepaticojejunostomy anastomosis was successful without requiring any modification or positional change for the patient or the endoscopist. The anastomotic site showed severe stenosis, with dilation of the intrahepatic bile ducts ( $\triangleright$ Fig. 3, \ Fig.4). After insertion of a guidewire across the anastomosis, we performed dilation to the stenosis using the Soehendra stent retriever (Cook Medical Inc., Bloomington, Indiana, USA). Then, a $7 \mathrm{Fr}$ plastic stent was placed in the intrahepatic bile duct ( $\triangleright$ Fig.5, $>$ Video 1 ).

The procedure time was 24 minutes. No adverse event occurred. The patient's symptoms and laboratory data rapidly improved after the endoscopic treatment.

For situs inversus, ERCP is challenging, and it has been suggested that alteration of patient and/or endoscopist positioning is warranted $[1,2]$. However, there are no reports of patients with situs in-

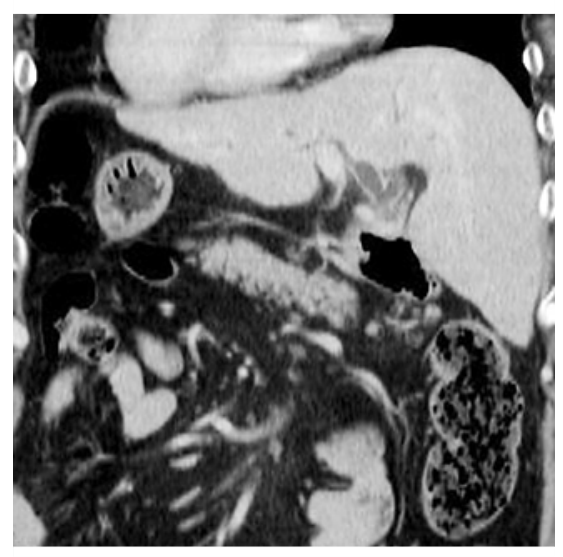

Fig. 1 Abdominal computed tomography revealed bile duct dilatation with stenosis at the hepaticojejunostomy anastomosis, with left-right transposition of all viscera.

versus undergoing balloon enteroscopyassisted ERCP. In the present case, the procedure could be performed using the same technique as that used in patients with normal anatomy. Even in situs inversus, balloon enteroscopy-assisted ERCP

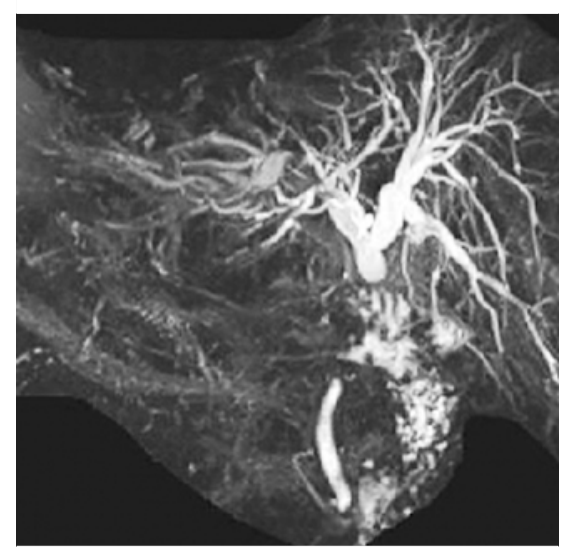

Fig. 2 Magnetic resonance cholangiopancreatography revealed intrahepatic bile duct dilatation.

can be performed in the usual manner for patients with surgically altered anatomy.

Endoscopy_UCTN_Code_TTT_1AR_2AG

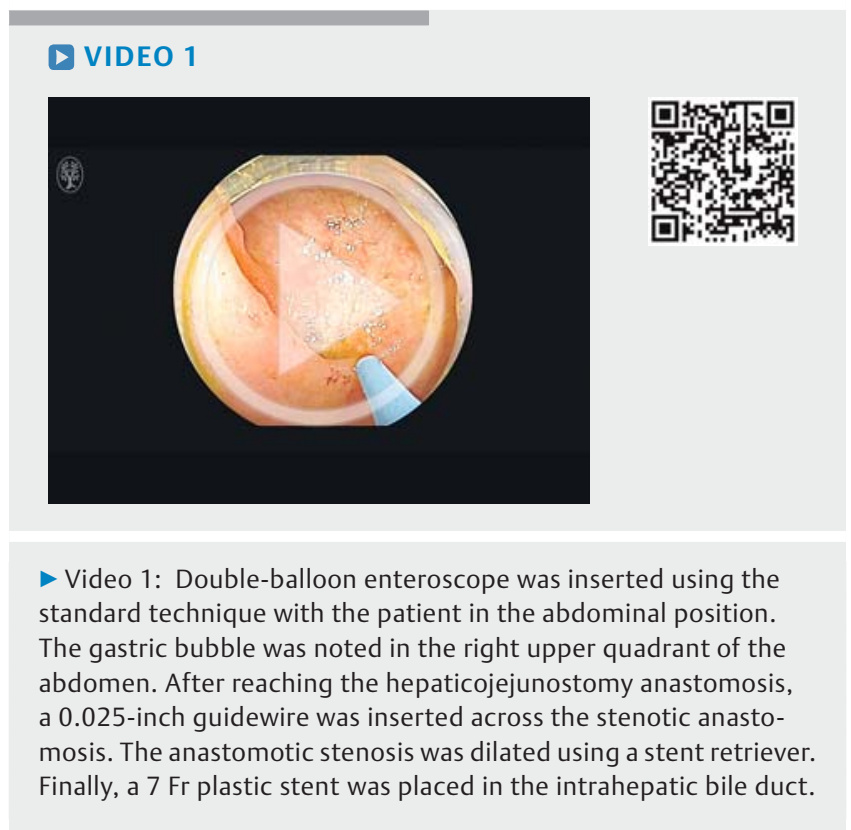




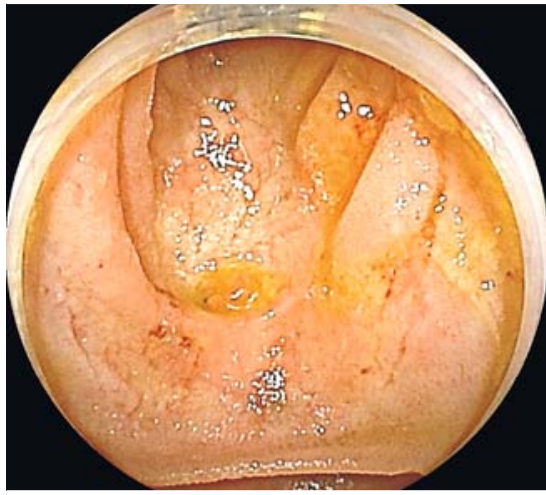

- Fig. 3 Endoscopic view showing severe stenosis at the hepaticojejunostomy anastomosis.

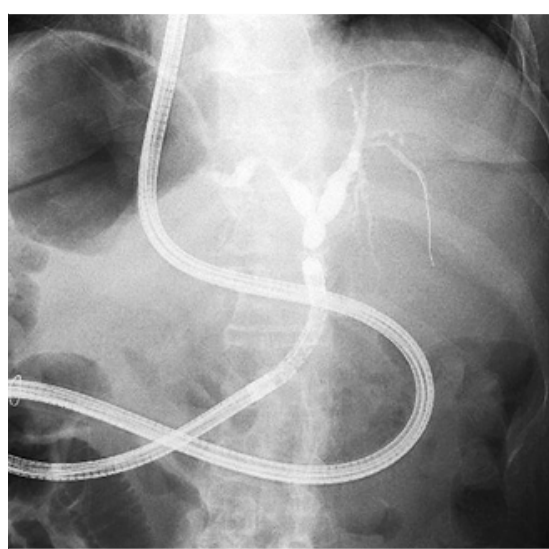

- Fig.4 Cholangiography showed dilation of the intrahepatic bile duct and stenosis of the anastomotic site.

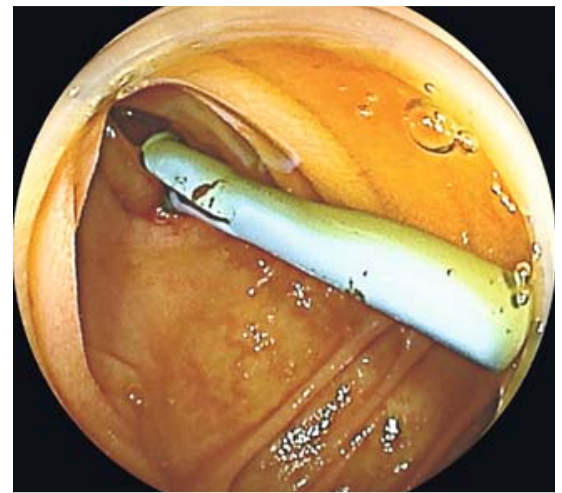

- Fig. 5 A 7 Fr plastic stent was placed in the intrahepatic bile duct.

\section{Competing interests}

None

The Authors

Tadahisa Inoue, Takaya Yamamoto,

Norimitsu Ishii, Yuji Kobayashi,

Kiyoaki Ito, Masashi Yoneda

Department of Gastroenterology, Aichi Medical

University School of Medicine, Nagakute, Japan
Corresponding author

Tadahisa Inoue, MD

Department of Gastroenterology, Aichi Medical University School of Medicine,

1-1 Yazakokarimata, Nagakute, Aichi 4801195, Japan

Fax: +81-561-633208

tinoue-tag@umin.ac.jp

\section{References}

[1] Hu Y, Zeng H, Pan XL et al. Therapeutic endoscopic retrograde cholangiopancreatography in a patient with situs inversus viscerum. World J Gastroenterol 2015; 24: $5744-5748$

[2] Garcia-Fernandez FJ, Infantes JM, Torres Y et al. ERCP in complete situs inversus viscerum using a "mirror image" technique. Endoscopy 2010; 42: E316-317

\section{Bibliography}

DOI http://dx.doi.org/10.1055/s-0042-121011

Endoscopy 2017; 49: E46-E47

(c) Georg Thieme Verlag KG

Stuttgart · New York

ISSN 0013-726X 